\title{
(Lineare) Regression/Korrelation
}

\author{
St. Lange ${ }^{1}$, R. Bender ${ }^{2}$ \\ 1 Abteilung für Medizinische Informatik, Biometrie und Epidemiologie der Ruhr-Universität Bochum \\ 2 Fakultät für Gesundheitswissenschaften, AG Epidemiologie und medizinische Statistik, Universität Bielefeld
}

Neben der univariaten, das heißt auf ein einzelnes Merkmal bezogenen Analyse von Daten aus einer klinischen Studie, ist man häufig daran interessiert, den Zusammenhang zwischen zwei (bivariat) oder mehreren (multivariat) Variablen zu betrachten. Bei Betrachtung von zwei quantitativen Merkmalen bietet sich als anschauliche, graphische Darstellungsweise die Punktwolke an, bei der die Wertepaare durch einen Punkt in einem Koordinatensystem abgebildet werden (Abb.1). Damit wird sofort visuell erfassbar, ob überhaupt ein Zusammenhang besteht, und wenn ja, wie stark er ist. Tab.1 enthält die Werte für den systolischen Blutdruck und das Körpergewicht von 24 zufällig ausgewählten Patienten einer dermatologischen Ambulanz. Abb.1 zeigt die dazugehörige Punktwolke, die einen recht deutlichen Zusammenhang zwischen den beiden Merkmalen erkennen lässt.

Eine Möglichkeit, den Zusammenhang zwischen Merkmalen statistisch zu beschreiben, bietet die Regressionsanalyse. Bei der einfachen, linearen Regression, bei der Analyse von zwei quantitativen Merkmalen, erfolgt anhand einer Geradengleichung die Vorhersage von Werten einer abhängigen Variablen aus den Werten einer als unabhängig angesehenen Variablen; es wird also ein Modell verwendet. Modelle können die Realität meist nur unvollkommen beschreiben, aber das lineare Modell hat sich für viele medizinische Anwendungen als sinnvoll und hilfreich erwiesen. Die Angemessenheit lässt sich häufig bereits bei der visuellen Betrachtung der Punktwolke beurteilen.

Ähnlich wie der Mittelwert im univariaten Fall einen typischen Wert der Stichprobe für das betrachtete Merkmal repräsentiert (2), liefert die Regressionsgerade einen typischen Wert der abhängigen Variablen bei gegebenem Wert der unabhängigen. Das Stichwort der "Vorhersage» macht deutlich, dass bei der Regression die Richtung des Zusammenhangs üblicherweise vorgegeben wird, das heißt es können schon a priori sinnvoll eine abhängige (Outcome) Variable, deren Werte vorhergesagt werden sollen, und eine unabhängige (Prädiktor) Variable definiert werden. Für die Punktwolke wird als Konvention die abhängige Variable zumeist auf der Ordinate (y-Achse) und die unabhängige Variable auf der Abszisse ( $\mathrm{x}$-Achse) abgebildet.

Eine Geradengleichung benötigt zwei Parameter: Zum einen die Steigung der Geraden, die angibt, um wie viel die Werte der abhängigen Variable steigen oder fallen, wenn sich die unabhängige Variable um eine Einheit verändert, und zum zweiten der Achsenabschnitt, der das Basisniveau der abhängigen Variable angibt, wenn also die unabhängige Variable

Dtsch. Med. Wschr. 2001; 126: T33-T35

(c) Georg Thieme Verlag Stuttgart · New York

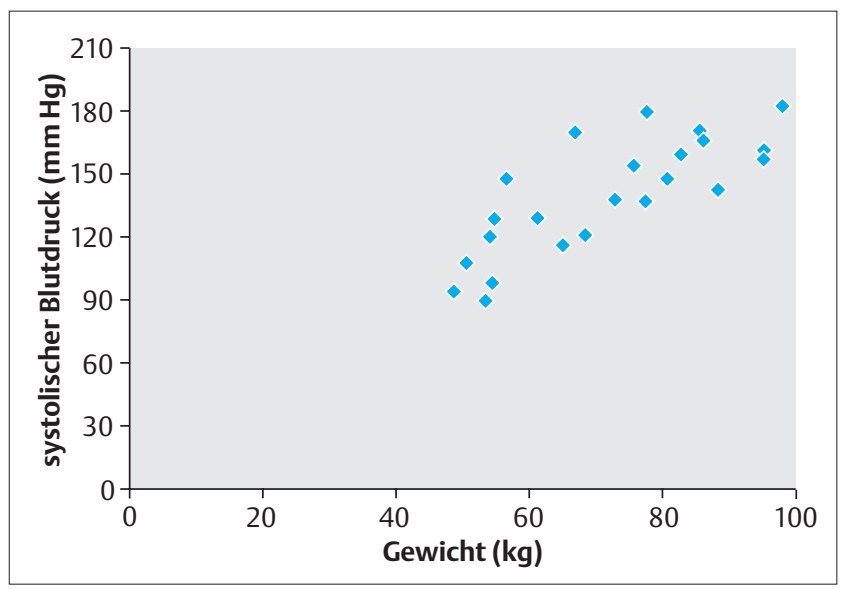

Abb.1 Punktwolke für den Zusammenhang zwischen Körpergewicht $(\mathrm{kg})$ und systolischen Blutdruck $(\mathrm{mm} \mathrm{Hg})$ von 24 zufällig ausgewählten Patienten einer dermatologischen Ambulanz.

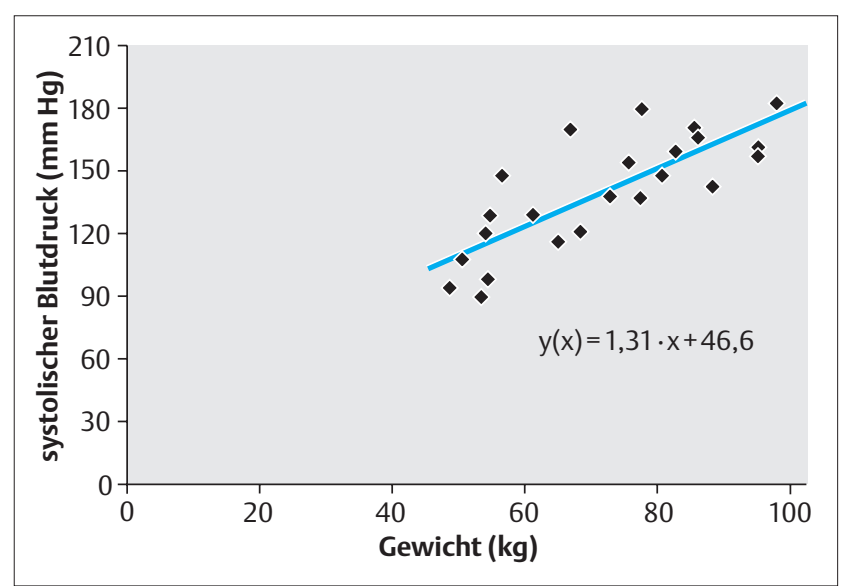

Abb. 2 Punktwolke mit Regressionsgerade und Regressionsgleichung für den Zusammenhang zwischen Körpergewicht $(\mathrm{kg})$ und systolischen Blutruck $(\mathrm{mm} \mathrm{Hg})$. Das Körpergewicht ist die unabhängige (Predictor), der systolische Blutdruck die abhängige (Outcome) Variable.

den Wert Null annimmt. Die Steigung der Geraden wird als Regressionskoeffizient bezeichnet.

In Abb. $\mathbf{2}$ ist die Regressionsgerade mit der entsprechenden Regressionsgleichung für die Daten aus Tab.1 dargestellt. Es erscheint plausibel, den Blutdruck in Abhängigkeit vom Gewicht und nicht umgekehrt zu betrachten. Die Geradengleichung zeigt an, dass der Wert des systolischen Blutdrucks im Mittel um ca. 1,31 mm Hg ansteigt, wenn der Wert des Körpergewichts um $1 \mathrm{~kg}$ zunimmt. Bei einer $70 \mathrm{~kg}$ schweren Per- 
Tab. 1 Körpergewicht $(\mathrm{kg})$ und systolische Blutdruckwerte $(\mathrm{mm} \mathrm{Hg})$ von 24 zufällig ausgewählten Patienten einer dermatologischen Ambulanz.

\begin{tabular}{|c|c|c|}
\hline Patientennummer & Körpergewicht (kg) & $\begin{array}{l}\text { systolischer Blutdruck } \\
(\mathrm{mm} \mathrm{Hg})\end{array}$ \\
\hline 1 & 54,5 & 128 \\
\hline 2 & 77,0 & 154 \\
\hline 3 & 78,5 & 180 \\
\hline 4 & 48,0 & 96 \\
\hline 5 & 90,0 & 142 \\
\hline 6 & 86,5 & 170 \\
\hline 7 & 54,6 & 122 \\
\hline 8 & 61,0 & 130 \\
\hline 9 & 66,0 & 118 \\
\hline 10 & 54,0 & 98 \\
\hline 11 & 85,0 & 172 \\
\hline 12 & 80,0 & 149 \\
\hline 13 & 80,5 & 150 \\
\hline 14 & 96,7 & 181 \\
\hline 15 & 68,0 & 170 \\
\hline 16 & 50,0 & 109 \\
\hline 17 & 71,5 & 140 \\
\hline 18 & 55,0 & 150 \\
\hline 19 & 78,5 & 139 \\
\hline 20 & 94,5 & 157 \\
\hline 21 & 68,7 & 121 \\
\hline 22 & 97,2 & 160 \\
\hline 23 & 53,0 & 91 \\
\hline 24 & 84,0 & 161 \\
\hline
\end{tabular}

son ist mit einem Blutdruck von $70 \times 1,31+46,6 \approx 138 \mathrm{~mm} \mathrm{Hg}$ zu rechnen. Die am besten "passende" Regressionsgerade wird durch ein besonderes statistisches Schätzverfahren - die Kleinste-Quadrate-Methode - gefunden, und zwar ist es diejenige Gerade, bei der die Summe der quadrierten (vertikalen) Abstände zwischen den einzelnen Punkten und der Geraden minimal wird.

kurzgefasst: Mit der Regression lässt sich der Zusammenhang zwischen einer abhängigen und einer oder mehreren unabhängigen Variablen darstellen. Die Regressionsgleichung liefert den Wert der abhängigen Variable, wenn die unabhängige bekannt ist.

Für eine weitere Quantifizierung des beobachteten Zusammenhangs zwischen den Merkmalen ist das Bestimmtheitsmaß $\left(R^{2}\right)$ ein sehr anschaulicher Parameter. Hierfür muss man sich zunächst vergegenwärtigen, dass die Werte der abhängigen Variable - im Beispiel die Blutdruckwerte - bei univariater Betrachtung um ihren Mittelwert "streuen«; diese Streuung wird als Summe der quadratischen Abweichungen (der Einzelwerte von ihrem Mittelwert) ausgedrückt (3). Die Blutdruckwerte streuen auch um die Regressionsgerade, aber in einem geringeren Ausmaß als um ihren Mittelwert. Das Bestimmtheitsmaß bezeichnet nun den Anteil, um den die Variabilität der abhängigen Variable durch die Regression, also durch die zusätzliche Betrachtung der unabhängigen Variab-
Tab. 2 Übersetzungen (deutsch - englisch)

$\begin{array}{ll}\text { Kleinste-Quadrate-Methode } & \begin{array}{l}\text { least-square-method } \\ \text { Vorhersage }\end{array} \\ \text { Bestimmtheitsmaß }\left(\mathrm{R}^{2}\right) & \text { coefficiont of determination } \\ \text { (Un)abhängige Variable } & \text { (in)dependent variable } \\ \text { Korrelationskoeffizient } & \text { correlation coefficient } \\ \text { Regression } & \text { regression } \\ \text { Regressionsgerade } & \text { regression } \\ \text { (-koeffizient) } & \text { line (coefficient) } \\ \text { Punktwolle } & \text { scatter plot }\end{array}$

le, vermindert wird. Als Maß für die Streuung um die Regressionsgerade wird wieder die Summe von Abweichungsquadraten (der Einzelwerte von der Regressionsgerade) verwendet. Im Beispiel ergibt sich ein Bestimmtheitsmaß von 0,62, also $62 \%$ der "rohen" Variabilität der Blutdruckwerte aus der Stichprobe kann durch das Körpergewicht der Patienten »erklärt» werden (unter Annahme des linearen Modells).

Ein weiteres Maß für die Quantifizierung des Zusammenhangs zwischen zwei (quantitativen) Merkmalen ist der Korrelationskoeffizient "ru. Der Absolutbetrag des Korrelationskoeffizienten nach Pearson ist einfach die Wurzel aus dem Bestimmtheitsmaß: $|r|=\sqrt{ } \mathrm{R}^{2} . r$ kann Werte zwischen -1 (negativer Zusammenhang) und +1 (positiver Zusammenhang) annehmen. Das Vorzeichen von $r$ ist dasselbe wie das des Regresionskoeffizienten. Ein Korrelationskoeffizient von Null bedeutet, dass kein linearer Zusammenhang besteht. Für das Beispiel ergibt sich $|r|=\sqrt{ } 0,62 \approx 0,79$. Anstelle des Korrelationskoeffizienten nach Pearson kann auch der Rangkorrelationskoeffizient nach Spearman berechnet werden. Er basiert, wie der Name andeutet, nicht auf den Messwerten, sondern auf den Rangzahlen, die die Messwerte in der sortierten Stichprobe einnehmen. Er ist in gleicher Weise zu interpretieren wie der Korrelationskoeffizient nach Pearson und wird insbesondere bei der Betrachtung von Scores benutzt.

Der Korrelationskoeffizient ist eines der am häufigsten, leider oft auch fälschlich eingesetzten Maße in der medizinischen Statistik. Deshalb soll auf folgende, für eine adäquate Interpretation zu beachtende Punkte hingewiesen werden:

- Der Korrelationskoeffizient, genauso wie die Regressionsgerade, liefert keine Aussage über einen kausalen Zusammenhang.

- Der Wert des Korrelationskoeffizienten kann sehr stark durch Extremwerte beeinflusst werden. Das ist leicht nachzuvollziehen, da Extremwerte die Varianz eines Merkmals stark erhöhen, und dann durch die Regression sehr viel von dieser Varianz "erklärt» werden kann.

- Die gemeinsame Betrachtung von zwei sehr unterschiedlichen Gruppen kann zu einer hohen Korrelation zwischen Merkmalen führen, obwohl innerhalb jeder Gruppe nur eine geringe oder gar keine Korrelation zwischen den Merkmalen besteht (Heterogenitätskorrelation).

- Der Korrelationskoeffizient ist kein Maß für Übereinstimmung! Seine Verwendung beim Vergleich zweier Messverfahren ist daher für sich allein nicht aussagefähig und häufig nicht adäquat $(1,4)$. Ein Korrelationskoeffizient nahe 1 wird auch dann erreicht, wenn zum 
Beispiel beim Vergleich zweier Verfahren zur Blutzuckermessung das eine Verfahren doppelt so hohe Werte liefert wie das andere.

kurzgefasst: Der Korrelationskoeffizient $\mathrm{R}$ zeigt den linearen Zusammenhang zwischen 2 Variablen. Er kann Werte zwischen -1 und +1 einnehmen. Der Korrelationskoeffizient dient NICHT der Darstellung von kausalen Zusammenhängen oder Übereinstimmungen.

Tab.2 zeigt wieder die Übersetzungen für die Interpretation englischsprachiger Studien.

\section{Literatur}

1 Bland JM, Altman DG. Statistical methods for assessing agreement between two methods of clinical measurement. Lancet 1986; I: 307-310

2 Lange S, Bender R. Median oder Mittelwert? Dtsch med Wschr 2001; 126: T25-T26

3 Lange S, Bender R. Variablitätsmaße. Dtsch med Wschr 2001; 126 : T29-T30

4 Richter K, Lange S. Methoden der Diagnoseevaluierung. Internist 1997; 38: 325-336

\section{Korrespondenz}

Dr. Stefan Lange

Abteilung für Medizinische Informatik, Biometrie und

Epidemiologie

Ruhr-Universität

Universitätsstraße 150

44780 Bochum

E-Mail: stefan.f.lange@ruhr-uni-bochum.de 